\title{
ASYMPTOTICS FOR ORTHOGONAL POLYNOMIALS AND Christoffel FUnCTIONS ON A BALL
}

\author{
Yuan Xu
}

\begin{abstract}
We derive the asymptotics for a sum of squares of orthonormal polynomials in several variables with respect to the weight function $\left(1-|\mathbf{x}|^{2}\right)^{\mu-1 / 2}$ on the unit ball in $\mathbb{R}^{d}$; the asymptotics for the corresponding Christoffel functions proved in [3] by Bos follow as a consequence. We also obtain an upper bound for the Christoffel functions with respect to a large class of radial weight functions on the unit ball.
\end{abstract}

\section{Introduction}

Let $\mathbb{N}_{0}$ be the set of nonnegative integers. For $\alpha=\left(\alpha_{1}, \ldots, \alpha_{d}\right) \in \mathbb{N}_{0}^{d}$, we write $|\alpha|_{1}=\alpha_{1}+\cdots+\alpha_{d}$. For $\alpha \in \mathbb{N}_{0}^{d}$ and $\mathbf{x}=\left(x_{1}, \ldots, x_{d}\right) \in \mathbb{R}^{d}$, we write $\mathbf{x}^{\alpha}=x_{1}^{\alpha_{1}} \cdots x_{d}^{\alpha_{d}}$, monomials of degree $|\alpha|_{1}$. Let $\Pi^{d}$ be the space of polynomials in $d$ variables and $\Pi_{n}^{d}$ be the subspace of polynomials of degree at most $n$. For a nonnegative weight function $W$ on a compact set $\Omega \subset \mathbb{R}^{d}$, we can use the Gram-Schmidt orthogonalization process on the sequence of monomials to obtain a sequence of orthonormal polynomials which forms a basis of $\Pi^{d}$. The Gram-Schmidt process depends on the ordering of the monomials; different orderings will lead to different sequences of orthonormal bases. The nonuniqueness of the bases is one of the essential difficulties in dealing with orthogonal polynomials in several variables. We denote by $\left\{P_{\alpha}^{n}\right\}, \alpha \in \mathbb{N}_{0}^{d},|\alpha|_{1}=n$, and $n \in \mathbb{N}_{0}$, one family of orthonormal polynomials, where the superscript $n$ means that $P_{\alpha}^{n} \in \Pi_{n}^{d}$. Setting $r_{n}^{d}=\left(\begin{array}{c}n+d-1 \\ n\end{array}\right)$, which is the cardinal of the set $\left\{\alpha \in \mathbb{N}_{0}^{d}\right.$ $\left.:|\alpha|_{1}=n\right\}$, we arrange the polynomials $P_{\alpha}^{n},|\alpha|_{1}=n$, according to the lexicographical order: $P_{\alpha_{1}}, \ldots, P_{\alpha_{r_{n}^{d}}}$, where $\alpha_{i} \in \mathbb{N}_{0}^{d}$. A useful vector notation

$$
\mathbb{P}_{n}=\left(P_{\alpha_{1}}^{n}, P_{\alpha_{2}}^{n}, \ldots, P_{\alpha_{r_{n}^{d}}}^{n}\right)^{T}
$$

is introduced in [9]. The nonuniqueness of the orthonormal basis can be seen easily from the fact that for any orthogonal matrix $Q_{n}$ the components of $Q_{n} \mathbb{P}_{n}$ are also orthonormal polynomials. Moreover, any two sequences of orthonormal polynomials are related in this way. It turns out that many results concerning orthogonal polynomials in several variables can be stated independent of the choice of the bases, which is the starting point of our recent investigations in [9] and a number of subsequent papers (cf. [10] and the references therein). In particular, the $n$-th reproducing kernel, defined by

$$
\mathbf{K}_{n}(\mathbf{x}, \mathbf{y})=\sum_{k=0}^{n} \sum_{|\alpha|_{1}=k} P_{\alpha}^{k}(\mathbf{x}) P_{\alpha}^{k}(\mathbf{y})=\sum_{k=0}^{n} \mathbb{P}_{k}^{T}(\mathbf{x}) \mathbb{P}_{k}(\mathbf{y})
$$

Received September 25, 1995, revised February 12, 1996.

1991 Mathematics Subject Classification: Primary 41A63, 33C50; Secondary 42C05.

Key words and phrases: orthogonal polynomials in several variables, Jacobi weight on the unit ball, asymptotics, Christoffel function. 
is independent of the choice of the bases. In this regard, a more basic quantity is $\mathbb{P}_{n}^{T}(\mathbf{x}) \mathbb{P}_{n}(\mathbf{y})$. One main result of this paper is the asymptotics of $\mathbb{P}_{n}^{T}(\mathbf{x}) \mathbb{P}_{n}(\mathbf{x})$ for the weight functions $W_{\mu}(\mathbf{x})=w_{\mu}\left(1-|\mathbf{x}|^{2}\right)^{\mu-1 / 2}$ defined on the unit ball in $\mathbb{R}^{d}$ where $w_{\mu}$ is a constant chosen so that $W_{\mu}$ has integral 1 . We shall prove that for $d \geq 2$,

$$
\lim _{n \rightarrow \infty} \frac{1}{\left(\begin{array}{c}
n+d-1 \\
n
\end{array}\right)} \mathbb{P}_{n}^{T}(\mathbf{x}) \mathbb{P}_{n}(\mathbf{x})=\frac{W_{0}(\mathbf{x})}{W_{\mu}(\mathbf{x})}, \quad 0<|\mathbf{x}|<1,
$$

and results will be stated for $|\mathbf{x}|=0$ and 1 as well. It is well-known that such a limit does not exist for $d=1$, where the orthogonal polynomials are the classical ultraspherical polynomials.

For $d=1$, limit relations of this type have been proved for the function $\mathbf{K}_{n}(\mathbf{x}, \mathbf{x})$ and the result often is stated in terms of the inverse of $\mathbf{K}_{n}(\mathbf{x}, \mathbf{x})$, called the Christoffel function, which plays an important role in the theory of orthogonal polynomials in one variable (cf. [7]). The Christoffel function satisfies an important property that we state below for $d \geq 1$,

$$
\Lambda_{n}(\mathbf{x}):=\left[\mathbf{K}_{n}(\mathbf{x}, \mathbf{x})\right]^{-1}=\min _{P(\mathbf{x})=1, P \in \Pi_{n}^{d}} \int_{\Omega} P^{2}(\mathbf{y}) W(\mathbf{y}) d \mathbf{y} .
$$

The asymptotics of the Christoffel function for $d=1$ have been established for general measures (weight functions) supported on $[-1,1]$ (cf. [7]). For $d>1$, however, little seems to be known in general (cf. [11]; we should mention that the notation $\mathbf{K}_{n}$ in [11] corresponds to $\mathbf{K}_{n-1}$ here). For the weight function $W_{\mu}$, the asymptotics of $\mathbf{K}_{n}(\mathbf{x}, \mathbf{x})$ have been established recently by Bos [3]. His proof depends heavily on (1.4). Since $\mathbf{K}_{n}(\mathbf{x}, \mathbf{x})$ is just a sum of $\mathbb{P}_{k}^{T}(\mathbf{x}) \mathbb{P}_{k}(\mathbf{x})$ 's, we will deduce its asymptotics from those of (1.3). Our proof depends on a compact formula for $\mathbb{P}_{n}^{T}(\mathbf{x}) \mathbb{P}_{n}(\mathbf{x})$, which we proved recently and used to study the summability of the Fourier orthogonal series [12]. The compact formula takes a particularly simple form for the radial Chebyshev weight function $W_{0}(\mathbf{x})$, which allows us to establish the limit

$$
\limsup _{n \rightarrow \infty}\left(\begin{array}{c}
n+d \\
n
\end{array}\right) \Lambda_{n}(\mathbf{x}) \leq \frac{W_{0}(\mathbf{x})}{W(\mathbf{x})}, \quad|\mathbf{x}|<1
$$

for a large family of radial weight functions $W$ on $\mathbb{R}^{d}$.

The paper is organized as follows. In the next section, we fix notation and recall the necessary preliminaries. The result on the asymptotics of $\mathbb{P}_{n}^{T}(\mathbf{x}) \mathbb{P}_{n}(\mathbf{x})$ for $W_{\mu}$ is stated and proved in Section 3. The asymptotics of the Christoffel functions are presented in Section 4. In Section 5, we deduce several additional properties of orthogonal polynomials with respect to $W_{\mu}$ that are relevant and seem to be of independent interests.

\section{Notation and basic formulae}

Throughout this paper, we use $B^{d}$ to denote the unit ball in $\mathbb{R}^{d}$ and $S^{d-1}$ to denote the unit sphere in $\mathbb{R}^{d}$; that is,

$$
B^{d}=\left\{\mathbf{x} \in \mathbb{R}^{d}:|\mathbf{x}| \leq 1\right\} \quad \text { and } \quad S^{d-1}=\left\{\mathbf{x} \in \mathbb{R}^{d}:|\mathbf{x}|=1\right\}
$$

where the notation $|\cdot|$ stands for the Euclidean norm $|\mathbf{x}|^{2}=\sqrt{x_{1}^{2}+\cdots+x_{d}^{2}}$. We also write $\mathbf{x} \cdot \mathbf{y}=x_{1} y_{1}+\cdots+x_{d} y_{d}$ for the standard inner product of $\mathbb{R}^{d}$. We use $\omega_{d-1}$ to denote the surface area of $S^{d-1}$; it is known that $\omega_{d-1}=2 \pi^{d / 2} / \Gamma(d / 2)$. The weight function that we will deal with in this paper is the normalized function

$$
W_{\mu}(\mathbf{x})=W_{\mu, d}(\mathbf{x})=w_{\mu}\left(1-|\mathbf{x}|^{2}\right)^{\mu-\frac{1}{2}}, \quad \mu \geq 0, \quad \mathbf{x} \in B^{d},
$$


where $w_{\mu}$ is a constant chosen so that the integral of $W_{\mu}$ is 1 :

$$
w_{\mu}=w_{\mu, d}=\frac{2}{\omega_{d-1}} \frac{\Gamma\left(\mu+\frac{d+1}{2}\right)}{\Gamma\left(\mu+\frac{1}{2}\right) \Gamma\left(\frac{d}{2}\right)}=\frac{\Gamma\left(\mu+\frac{d+1}{2}\right)}{\pi^{d / 2} \Gamma\left(\mu+\frac{1}{2}\right)} .
$$

For $d=1$, the orthogonal polynomials with respect to the weight function $W_{\mu}$ are the ultraspherical polynomials, customarily denoted by $C_{n}^{(\mu)}$, which is why we use the exponent $\mu-(1 / 2)$ instead of $\mu$ in the definition of $W_{\mu}$. We denote orthonormal polynomials with respect to $W_{\mu}$ by $P_{\alpha}^{n,(\mu)}$ and $\mathbb{P}_{n}$ with respect to $W_{\mu}$ by $\mathbb{P}_{n}^{(\mu)}$, and denote the reproducing kernel by $\mathbf{K}_{n}^{(\mu)}(\cdot, \cdot)$.

One family of orthonormal polynomials with respect to $W_{\mu}$ can be given explicitly using the ultraspherical polynomials. For $d=2$, they are given in $[6,(3.8)$, p. 449] by

$$
P_{k}^{n,(\mu)}(x, y)=\left[h_{k, 2}^{n}\right]^{-\frac{1}{2}} C_{n-k}^{(\lambda+k)}(x)\left(1-x^{2}\right)^{k / 2} C_{k}^{\left(\lambda-\frac{1}{2}\right)}\left(y\left(1-x^{2}\right)^{-\frac{1}{2}}\right), \quad 0 \leq k \leq n,
$$

where $h_{k, 2}^{n}$ are constants chosen so that $P_{k}^{n,(\mu)}$ are normalized. In this case, it is more convenient to index the polynomials by $k$ instead of $\alpha=(k, n-k)$. We follow the standard normalization for the ultraspherical polynomials as in [8, p. 80]. They are orthogonal with respect to $\left(1-x^{2}\right)^{\mu-1 / 2}$ on $[-1,1]$, and they satisfy

$$
\int_{-1}^{1}\left[C_{n}^{(\lambda)}(x)\right]^{2}\left(1-x^{2}\right)^{\lambda-\frac{1}{2}}=2^{1-2 \lambda} \pi[\Gamma(\lambda)]^{-2} \frac{\Gamma(n+2 \lambda)}{(n+\lambda) \Gamma(n+1)}, \quad \lambda>-\frac{1}{2}, \quad \lambda \neq 0
$$

where, for $\lambda=0$, the above relation holds under the limit relation

$$
\lim _{\lambda \rightarrow 0} \frac{\lambda+n}{\lambda} C_{n}^{(\lambda)}(\cos \theta)= \begin{cases}1, & \text { for } n=0, \\ 2 \cos n \theta, & \text { for } n=1,2, \ldots\end{cases}
$$

For this paper, we do not need the explicit formula for $P_{\alpha}^{n,(\mu)}$ (cf. [12]). What we need is the following compact formula for $\left[\mathbb{P}_{n}^{(\mu)}(\mathbf{x})\right]^{T} \mathbb{P}_{n}^{(\mu)}(\mathbf{y})$, which we proved in [12] with the help of the explicit formula for $P_{\alpha}^{n,(\mu)}$.

Theorem 2.1. For $W_{\mu}$ on $B^{d}, \mu>0$,

$$
\begin{aligned}
{\left[\mathbb{P}_{n}^{(\mu)}(\mathbf{x})\right]^{T} \mathbb{P}_{n}^{(\mu)}(\mathbf{y})=\frac{n+\mu+\frac{d-1}{2}}{\mu+\frac{d-1}{2}} \int_{0}^{\pi} C_{n}^{\left(\mu+\frac{d-1}{2}\right)}\left(\mathbf{x} \cdot \mathbf{y}+\sqrt{1-|\mathbf{x}|^{2}} \sqrt{1-|\mathbf{y}|^{2}} \cos \psi\right) } \\
\times(\sin \psi)^{2 \mu-1} d \psi / \int_{0}^{\pi}(\sin \psi)^{2 \mu-1} d \psi, \quad \mathbf{x}, \mathbf{y} \in B^{d}
\end{aligned}
$$

and, for $\mu=0$,

$$
\begin{aligned}
{\left[\mathbb{P}_{n}^{(0)}(\mathbf{x})\right]^{T} \mathbb{P}_{n}^{(0)}(\mathbf{y})=} & \frac{n+\frac{d-1}{2}}{d-1}\left[C_{n}^{\left(\frac{d-1}{2}\right)}\left(\mathbf{x} \cdot \mathbf{y}+\sqrt{1-|\mathbf{x}|^{2}} \sqrt{1-|\mathbf{y}|^{2}}\right)\right. \\
& \left.+C_{n}^{\left(\frac{d-1}{2}\right)}\left(\mathbf{x} \cdot \mathbf{y}-\sqrt{1-|\mathbf{x}|^{2}} \sqrt{1-|\mathbf{y}|^{2}}\right)\right], \quad \mathbf{x}, \mathbf{y} \in B^{d} .
\end{aligned}
$$

The compact formulae (2.5) and (2.6) have been used to prove in [12] that the expansion of a continuous function in the Fourier orthogonal series with respect to $W_{\mu}$ is uniformly $(C, \delta)$ summable on the ball if and only if $\delta>\mu+(d-1) / 2$. That was proved by using the addition formula and the product formula satisfied by the ultraspherical polynomials [5, Vol. I, Sec. 3.15.1, (19) and (20)]. In fact, for $d=1$, the formula (2.5) reduces to the product formula for the ultraspherical polynomials 
which, in consideration of the normalization constants in $(2.1)$, states that ([5, Vol. I, Sec. $3.15 .1,(20)]$ or $[2$, p. $30,(4.10)])$

$$
\frac{C_{n}^{(\lambda)}(\cos \theta) C_{n}^{(\lambda)}(\cos \phi)}{C_{n}^{(\lambda)}(1)}=c_{\lambda} \int_{0}^{\pi} C_{n}^{(\lambda)}(\cos \theta \cos \phi+\sin \theta \sin \phi \cos \psi)(\sin \psi)^{2 \lambda-1} d \psi
$$

where $\lambda>0$ and

$$
c_{\lambda}^{-1}=\int_{0}^{\pi}(\sin \psi)^{2 \lambda-1} d \psi=\int_{-1}^{1}\left(1-t^{2}\right)^{\lambda-1} d t=w_{\lambda-\frac{1}{2}, 1}^{-1} .
$$

Moreover, using the limit (2.4) for $\mu \rightarrow 0$, the formula reduces to the well-known formula for cosine,

$$
\cos n \theta \cos n \phi=\frac{1}{2}[\cos n(\theta+\phi)+\cos n(\theta-\phi)] .
$$

Later in the paper, we will need the product formula (2.7) and the following connection formula of Gegenbauer (cf. [2, p. 59]),

$$
C_{n}^{(\lambda)}(x)=\sum_{k=0}^{\left[\frac{n}{2}\right]} \frac{\Gamma(\mu)(n-2 k+\mu) \Gamma(k+\lambda-\mu)}{\Gamma(\lambda) \Gamma(\lambda-\mu) k ! \Gamma(n-k+\mu+1)} C_{n-2 k}^{(\mu)}(x)
$$

Other properties of the Gegenbauer polynomials that we shall need are ([8, (4.7.3) and (4.7.4), p. 80])

$$
C_{n}^{(\lambda)}(1)=\left(\begin{array}{c}
n+2 \lambda-1 \\
n
\end{array}\right), \quad C_{n}^{(\lambda)}(-x)=(-1)^{n} C_{n}^{(\lambda)}(x) .
$$

We also will need the asymptotic formula for $C_{n}^{(\lambda)}$ from [8, Theorem 8.21.8, p. 196], which we state as

Lemma 2.2. For $\lambda>0, x=\cos \theta$,

$$
C_{n}^{(\lambda)}(x)=\frac{\Gamma\left(\lambda+\frac{1}{2}\right) \Gamma(n+2 \lambda) 2^{\lambda}}{\Gamma(2 \lambda) \Gamma\left(n+\lambda+\frac{1}{2}\right) \Gamma\left(\frac{1}{2}\right)}\left[\frac{1}{(\sin \theta)^{\lambda}} n^{-\frac{1}{2}} \cos \left((n+\lambda) \theta-\frac{\lambda \pi}{2}\right)+\mathcal{O}\left(n^{-\frac{3}{2}}\right)\right]
$$

for $0<\theta<\pi ;$ in particular,

$$
C_{n}^{(\lambda)}(x)=\mathcal{O}\left(n^{\lambda-1}\right), \quad 0<\theta<\pi,
$$

where the bound for the error term holds uniformly in $[\varepsilon, \pi-\varepsilon]$.

The asymptotic formula (2.10) is stated in [8] for the Jacobi polynomials, while we have incorporated here the normalization constants as in $[8$, p. 80, (4.7.1)]. The estimate (2.11) is derived by using the following asymptotic formula for the Gamma function [13, p. 77],

$$
\frac{\Gamma(n+\lambda+1)}{\Gamma(n+1)}=n^{\lambda}\left(1+\mathcal{O}\left(n^{-1}\right)\right), \quad \lambda>0 .
$$

To end this section, we note that by setting $\mathbf{y}=\mathbf{x}$ in the compact formula (2.5) and (2.6), it follows readily that the polynomial $\left[\mathbb{P}_{n}^{(\mu)}(\mathbf{x})\right]^{T} \mathbb{P}_{n}^{(\mu)}(\mathbf{x})$ is a radial function. So is the function $\mathbf{K}_{n}^{(\mu)}(\mathbf{x}, \mathbf{x})$, by (1.2). This allows us to define

$$
\left|\mathbb{P}_{n, d}^{(\mu)}\right|^{2}(r)=\left[\mathbb{P}_{n}^{(\mu)}(\mathbf{x})\right]^{T} \mathbb{P}_{n}^{(\mu)}(\mathbf{x}), \quad \mathbf{K}_{n, d}^{(\mu)}(r)=\mathbf{K}_{n}^{(\mu)}(\mathbf{x}, \mathbf{x}), \quad r=|\mathbf{x}|, \quad \mathbf{x} \in \mathbb{R}^{d} .
$$

We may omit the subscript $d$ when it will not cause any confusion. 


\section{Asymptotics of orthogonal polynomials}

We start with a simple but important result.

Theorem 3.1. For $\mu \geq 0$ and $d \geq 1$,

$$
\left|\left[\mathbb{P}_{n}^{(\mu)}(\mathbf{x})\right]^{T} \mathbb{P}_{n}^{(\mu)}(\mathbf{y})\right| \leq\left|\mathbb{P}_{n}^{(\mu)}\right|^{2}(1)=\frac{n+\mu+\frac{d-1}{2}}{\mu+\frac{d-1}{2}} C_{n}^{\left(\mu+\frac{d-1}{2}\right)}(1) \quad \mathbf{x}, \mathbf{y} \in B^{d}
$$

and

$$
\left|\mathbf{K}_{n}^{(\mu)}(\mathbf{x}, \mathbf{y})\right| \leq \mathbf{K}_{n}^{(\mu)}(1)=C_{n}^{\left(\mu+\frac{d+1}{2}\right)}(1)+C_{n-1}^{\left(\mu+\frac{d-1}{2}\right)}(1) \quad \mathbf{x}, \mathbf{y} \in B^{d} .
$$

In particular, both $\left|\mathbb{P}_{n}^{(\mu)}\right|(|\cdot|)$ and $\mathbf{K}_{n}^{(\mu)}(|\cdot|)$ attain their maxima on $B^{d}$ at the boundary. Proof. Since the absolute value of the ultraspherical polynomial $C_{n}^{(\lambda)}$ attains its maximum on $[-1,1]$ at the boundary, the inequality (3.1) is an immediate consequence of (2.5) and (2.6). Moreover, since $\left|\mathbb{P}_{n}^{(\mu)}\right|^{2}(1)=\left[\mathbb{P}_{n}^{(\mu)}(\mathbf{e})\right]^{T} \mathbb{P}_{n}^{(\mu)}(\mathbf{e})$ where $\mathbf{e}$ is any point on the boundary of $B^{d}$, we see that $\left|\mathbb{P}_{n}^{(\mu)}\right|^{2}(\cdot)$ attains its maximum on $B^{d}$ at the boundary. By $[8$, p. 83, (4.7.29)], we have

$$
\frac{k+\lambda}{\lambda} C_{k}^{(\lambda)}(x)=C_{k}^{(\lambda+1)}(x)-C_{k-2}^{(\lambda+1)}(x), \quad k \geq 0,
$$

where $C_{-2}^{(\lambda+1)}=C_{-1}^{(\lambda+1)}=0$, from which it follows readily that

$$
\sum_{k=0}^{n} \frac{k+\lambda}{\lambda} C_{k}^{(\lambda)}(x)=C_{n}^{(\lambda+1)}(x)+C_{n-1}^{(\lambda+1)}(x) .
$$

Using this formula and (1.2), the inequality (3.2) follows from (3.1).

The asymptotics of $\left|\mathbb{P}_{n, d}^{(\mu)}\right|^{2}$ are stated in the following two theorems. The first one deals with the case $\mu=0$.

Theorem 3.2. For $W_{0, d}, d \geq 2$, the following limit holds

$$
\lim _{n \rightarrow \infty} \frac{1}{\left(\begin{array}{c}
n+d-1 \\
n
\end{array}\right)}\left|\mathbb{P}_{n, d}^{(0)}\right|^{2}(|\mathbf{x}|)= \begin{cases}1, & \text { if } 0<|\mathbf{x}|<1 \text { and } d \geq 3, \\
2, & \text { if }|\mathbf{x}|=1,\end{cases}
$$

while for $d=2$ and $0<|\mathbf{x}|<1$, the above limit does not exist; moreover,

$$
\left|\mathbb{P}_{2 n+1, d}^{(0)}\right|^{2}(0)=0, \quad \text { and } \quad \lim _{n \rightarrow \infty} \frac{1}{\left(\begin{array}{c}
2 n+d-1 \\
2 n
\end{array}\right)}\left|\mathbb{P}_{2 n, d}^{(0)}\right|^{2}(0)=2 .
$$

Proof. Setting $\mathbf{y}=\mathbf{x}$ in (2.6), we obtain that

$$
\left|\mathbb{P}_{n, d}^{(0)}\right|^{2}(|\mathbf{x}|)=\frac{n+\frac{d-1}{2}}{d-1}\left[C_{n}^{\left(\frac{d-1}{2}\right)}\left(2|\mathbf{x}|^{2}-1\right)+C_{n}^{\left(\frac{d-1}{2}\right)}(1)\right]
$$

From (2.11) and the fact that

$$
C_{n}^{\left(\frac{d-1}{2}\right)}(1)=\left(\begin{array}{c}
n+d-2 \\
n
\end{array}\right)=\frac{d-1}{n+d-1}\left(\begin{array}{c}
n+d-1 \\
n
\end{array}\right),
$$

the limit (3.4) follows easily; moreover, for $d=2$, it is readily seen that

$$
\frac{1}{\left(\begin{array}{c}
n+1 \\
n
\end{array}\right)}\left|\mathbb{P}_{n, d}^{(0)}\right|^{2}(|\mathbf{x}|)=\frac{n+\frac{1}{2}}{n+1}\left[1+C_{n}^{\left(\frac{1}{2}\right)}\left(2|\mathbf{x}|^{2}-1\right)\right]
$$

which does not have a limit for $0<|\mathbf{x}|<1$ according to (2.10). Finally, for $|\mathbf{x}|=0$, the equation (3.5) follows from the above formula and (2.9). 
We note that $\mathbb{P}_{2 n+1, d}(0)=0$ holds not only for $W_{0}$ but for every centrally symmetric weight function ([10, Theorem 3.1.2]).

Theorem 3.3. For $W_{\mu, d}, \mu>0$ and $d \geq 2$,

$$
\lim _{n \rightarrow \infty} \frac{1}{\left(\begin{array}{c}
n+d-1 \\
n
\end{array}\right)}\left|\mathbb{P}_{n, d}^{(\mu)}\right|^{2}(|\mathbf{x}|)= \begin{cases}\frac{W_{0}(\mathbf{x})}{W_{\mu}(\mathbf{x})}, & \text { if } 0<|\mathbf{x}|<1 \\
\infty, & \text { if }|\mathbf{x}|=1\end{cases}
$$

Moreover,

$$
\left|\mathbb{P}_{2 n+1, d}^{(\mu)}\right|^{2}(0)=0, \quad \text { and } \quad \lim _{n \rightarrow \infty} \frac{1}{\left(\begin{array}{c}
2 n+d-1 \\
2 n
\end{array}\right)}\left|\mathbb{P}_{2 n, d}^{(\mu)}\right|^{2}(0)=\frac{2 W_{0}(0)}{W_{\mu}(0)}
$$

Proof. Since $W_{\mu}$ is a radial function on $\mathbb{R}^{d}$, we also write $W_{\mu, d}(r)=w_{\mu, d}\left(1-r^{2}\right)^{\mu-1 / 2}$. This slight abuse of notation will be used in this proof only. We always write $r=|\mathbf{x}|$. Setting $\mathbf{x}=\mathbf{y}$ in (2.5) leads to

$$
\left|\mathbb{P}_{n, d}^{(\mu)}\right|^{2}(r)=c_{\mu} \frac{n+\mu+\frac{d-1}{2}}{\mu+\frac{d-1}{2}} \int_{0}^{\pi} C_{n}^{\left(\mu+\frac{d-1}{2}\right)}\left(r^{2}+\left(1-r^{2}\right) \cos \psi\right)(\sin \psi)^{2 \mu-1} d \psi
$$

Using (3.3) with $\lambda=\mu+\frac{d-1}{2}$ as an iteration formula, we obtain

$$
C_{n}^{\left(\mu+\frac{d-1}{2}\right)}=\sum_{k=0}^{\left[\frac{n}{2}\right]} \frac{n-2 k+\mu+\frac{d-3}{2}}{\mu+\frac{d-3}{2}} C_{n-2 k}^{\left(\mu+\frac{d-3}{2}\right)} .
$$

It then follows from (3.8) that

$$
\left|\mathbb{P}_{n, d}^{(\mu)}\right|^{2}(r)=\frac{n+\mu+\frac{d-1}{2}}{\mu+\frac{d-1}{2}} \sum_{k=0}^{\left[\frac{n}{2}\right]}\left|\mathbb{P}_{n-2 k, d-2}^{(\mu)}\right|^{2}(r) .
$$

This formula allows us to do a dimension reduction in proving (3.6). Indeed, it is known that if $b_{n} \rightarrow \infty$ as $n \rightarrow \infty$, then

$$
\lim _{n \rightarrow \infty} \frac{a_{n}}{b_{n}}=\lim _{n \rightarrow \infty} \frac{a_{n}-a_{n-1}}{b_{n}-b_{n-1}}
$$

(cf. [4, p. 414] - I'm grateful to a referee for providing this reference). Hence, assuming that (3.6) has been proved for $\left|\mathbb{P}_{n, d}^{(\mu)}\right|^{2}(r)$, we then can derive from (3.9) that

$$
\begin{aligned}
\lim _{n \rightarrow \infty} \frac{1}{\left(\begin{array}{c}
n+d+1 \\
n
\end{array}\right)}\left|\mathbb{P}_{n, d+2}^{(\mu)}\right|^{2}(r) & =\lim _{n \rightarrow \infty} \frac{n+\mu+\frac{d+1}{2}}{\mu+\frac{d+1}{2}} \frac{1}{\left(\begin{array}{c}
n+d+1 \\
n
\end{array}\right)} \sum_{k=0}^{\left[\frac{n}{2}\right]}\left|\mathbb{P}_{n-2 k, d}^{(\mu)}\right|^{2}(r) \\
& =\lim _{n \rightarrow \infty} \frac{d+1}{\mu+\frac{d+1}{2}} \frac{1}{\left(\begin{array}{c}
n+d \\
n
\end{array}\right)} \sum_{k=0}^{\left[\frac{n}{2}\right]}\left|\mathbb{P}_{n-2 k, d}^{(\mu)}\right|^{2}(r) \\
& =\lim _{n \rightarrow \infty} \frac{d+1}{\mu+\frac{d+1}{2}} \frac{\left|\mathbb{P}_{n, d}^{(\mu)}\right|^{2}(r)}{\left(\begin{array}{c}
n+d \\
n
\end{array}\right)-\left(\begin{array}{c}
n+d-2 \\
n-2
\end{array}\right)} \\
& =\frac{d+1}{2 \mu+d+1} \lim _{n \rightarrow \infty} \frac{\left|\mathbb{P}_{n, d}^{(\mu)}\right|^{2}(r)}{\left(\begin{array}{c}
n+d-1 \\
n
\end{array}\right)\left(1+\mathcal{O}\left(n^{-1}\right)\right)} \\
& =\frac{d+1}{2 \mu+d+1} \frac{w_{0, d}}{w_{\mu, d}} \frac{1}{\left(1-r^{2}\right)^{\mu}}=\frac{w_{0, d+2}}{w_{\mu, d+2}} \frac{1}{\left(1-r^{2}\right)^{\mu}}
\end{aligned}
$$


where, in the last step, we have used the fact that

$$
\frac{w_{0, d+2}}{w_{\mu, d+2}}=\frac{\Gamma\left(\frac{d+3}{2}\right) \Gamma\left(\mu+\frac{1}{2}\right)}{\Gamma\left(\frac{1}{2}\right) \Gamma\left(\mu+\frac{d+3}{2}\right)}=\frac{d+1}{2 \mu+d+1} \frac{w_{0, d}}{w_{\mu, d}} .
$$

Therefore, by induction, we only need to prove (3.6) for the cases $d=2$ and $d=3$.

By the connection formula (2.8) with $\lambda=\mu+(d-1) / 2$ and the product formula (2.7), it follows from (3.8) that

$$
\left|\mathbb{P}_{n}^{(\mu)}\right|^{2}(r)=\frac{n+\mu+\frac{d-1}{2}}{\mu+\frac{d-1}{2}} \sum_{k=0}^{\left[\frac{n}{2}\right]} b_{k, n} \frac{\left[C_{n-2 k}^{(\mu)}(r)\right]^{2}}{C_{n-2 k}^{(\mu)}(1)}
$$

with

$$
b_{k, n}=\frac{\Gamma(\mu)(n-2 k+\mu) \Gamma\left(k+\frac{d-1}{2}\right) \Gamma\left(n-k+\mu+\frac{d-1}{2}\right)}{\Gamma\left(\mu+\frac{d-1}{2}\right) \Gamma\left(\frac{d-1}{2}\right) k ! \Gamma(n-k+\mu+1)} .
$$

Using the Lemma 2.2, (2.12), and the identities (cf. [1, p. 256, (6.1.18)]),

$$
\Gamma\left(\frac{1}{2}\right)=\sqrt{\pi} \quad \Gamma(2 \mu)=\frac{2^{2 \mu-1}}{\sqrt{\pi}} \Gamma\left(\mu+\frac{1}{2}\right) \Gamma(\mu)
$$

we obtain, as $m \rightarrow \infty$,

$$
\frac{\left[C_{m}^{(\mu)}(r)\right]^{2}}{C_{m}^{(\mu)}(1)}=\frac{\Gamma\left(\frac{1}{2}\right) \Gamma\left(\mu+\frac{1}{2}\right)}{\Gamma(\mu)} \frac{2}{\pi} \frac{1}{m+\mu}\left[\frac{1}{(\sin \theta)^{2 \mu}} \cos ^{2}\left((m+\mu) \theta-\frac{\mu \pi}{2}\right)+\mathcal{O}\left(m^{-1}\right)\right]
$$

Therefore, from (3.11), we obtain that

$$
\begin{aligned}
& \left|\mathbb{P}_{n}^{(\mu)}\right|^{2}(r)=\frac{n+\mu+\frac{d-1}{2}}{\mu+\frac{d-1}{2}} \frac{\Gamma\left(\frac{1}{2}\right) \Gamma\left(\mu+\frac{1}{2}\right)}{\Gamma(\mu)} \frac{2}{\pi} \frac{1}{(\sin \theta)^{2 \mu}} \\
& \quad \times \sum_{k=0}^{\left[\frac{n}{2}\right]} \frac{b_{k, n}}{n-2 k+\mu} \cos ^{2}\left((n-2 k+\mu) \theta-\frac{\mu \pi}{2}\right)+\mathcal{O}(1) n \sum_{k=0}^{\left[\frac{n}{2}\right]} \frac{b_{k, n}}{(n-2 k+\mu)^{2}} .
\end{aligned}
$$

Using the half-angle formula for cosine, we see that to prove (3.6) for $0<|\mathbf{x}|<1$, it suffices to show that the following three limits hold true:

$$
\begin{gathered}
\lim _{n \rightarrow \infty} \frac{1}{\left(\begin{array}{c}
n+d-1 \\
n
\end{array}\right)} \frac{n+\mu+\frac{d-1}{2}}{\mu+\frac{d-1}{2}} \frac{\Gamma\left(\frac{1}{2}\right) \Gamma\left(\mu+\frac{1}{2}\right)}{\Gamma(\mu)} \frac{1}{\pi} \sum_{k=0}^{\left[\frac{n}{2}\right]} \frac{b_{k, n}}{n-2 k+\mu}=\frac{w_{0, d}}{w_{\mu, d}}, \\
\lim _{n \rightarrow \infty} \frac{n}{\left(\begin{array}{c}
n+d-1 \\
n
\end{array}\right)} \sum_{k=0}^{\left[\frac{n}{2}\right]} b_{k, n} \frac{\cos (2(n-2 k+\mu) \theta-\mu \pi)}{n-2 k+\mu}=0
\end{gathered}
$$

and

$$
\lim _{n \rightarrow \infty} \frac{n}{\left(\begin{array}{c}
n+d-1 \\
n
\end{array}\right)} \sum_{k=0}^{\left[\frac{n}{2}\right]} \frac{b_{k, n}}{(n-2 k+\mu)^{2}}=0 .
$$

Moreover, we only need to deal with the cases $d=2$ and $d=3$. It turns out that the case $d=3$ is easier than the case $d=2$. 
To prove (A), we denote the expression on the left-hand side of (A) by $J_{n, d}$. Setting $d=3$ in (3.12) leads to the fact that the summation in $J_{n, 3}$ is equal to $\sum 1$. Therefore, we have

$$
\lim _{n \rightarrow \infty} J_{n, 3}=\frac{1}{\pi} \frac{\Gamma\left(\frac{1}{2}\right) \Gamma\left(\mu+\frac{1}{2}\right)}{\Gamma(\mu+2)} \lim _{n \rightarrow \infty} \frac{(n+\mu+1)\left[\frac{n}{2}\right]}{\left(\begin{array}{c}
n+2 \\
n
\end{array}\right)}=\frac{\Gamma\left(\mu+\frac{1}{2}\right)}{\Gamma(\mu+2) \Gamma\left(\frac{1}{2}\right)}=\frac{w_{0,3}}{w_{\mu, 3}} .
$$

The case $d=2$ is not so simple as the above one; setting $d=2$ in (3.12) and using (2.12), we end up with

$$
\begin{aligned}
J_{n, 2} & =\frac{1}{\pi\left(\mu+\frac{1}{2}\right)} \sum_{k=0}^{\left[\frac{n}{2}\right]} \frac{\Gamma\left(k+\frac{1}{2}\right) \Gamma\left(n-k+\mu+\frac{1}{2}\right)}{\Gamma(k+1) \Gamma(n-k+\mu+1)} \\
& =\frac{1}{\pi\left(\mu+\frac{1}{2}\right)} \sum_{k=1}^{\left[\frac{n}{2}\right]} \frac{1}{\sqrt{k}}\left[1+\mathcal{O}\left(\frac{1}{k}\right)\right] \frac{1}{\sqrt{n-k}}\left[1+\mathcal{O}\left(\frac{1}{n-k}\right)\right] \\
& =\frac{1}{\pi\left(\mu+\frac{1}{2}\right)} \sum_{k=1}^{\left[\frac{n}{2}\right]} \frac{1}{\sqrt{k(n-k)}}+\mathcal{O}\left(\frac{\log n}{\sqrt{n}}\right) .
\end{aligned}
$$

We may assume that $n$ is even, say, $n=2 m$. Then, it follows from a change of summation index $k \mapsto m-k$ that

$$
\begin{aligned}
J_{n, 2} & =\frac{1}{\pi\left(\mu+\frac{1}{2}\right)} \sum_{k=1}^{m} \frac{1}{\sqrt{m^{2}-k^{2}}}+\mathcal{O}\left(\frac{\log n}{\sqrt{n}}\right) \\
& =\frac{1}{\pi\left(\mu+\frac{1}{2}\right)} \frac{1}{m} \sum_{k=1}^{m} \frac{1}{\sqrt{1-(k / m)^{2}}}+\mathcal{O}\left(\frac{\log n}{\sqrt{n}}\right) .
\end{aligned}
$$

The summation above is a Riemann sum for the integral of $\left(1-x^{2}\right)^{-1 / 2}$; we obtain that

$$
\lim _{n \rightarrow \infty} J_{n, 2}=\frac{1}{\pi\left(\mu+\frac{1}{2}\right)} \int_{0}^{1} \frac{1}{\sqrt{1-x^{2}}} d x=\frac{1}{2 \mu+1}=\frac{w_{0,2}}{w_{\mu, 2}} .
$$

Thus, we have proved the limit (A) completely.

To prove (B), we first observe that by the addition formula for the cosine function, it suffices to prove that for $0<\theta<\pi$, both

$$
\lim _{n \rightarrow \infty} \frac{n}{\left(\begin{array}{c}
n+d-1 \\
n
\end{array}\right)} \sum_{k=0}^{\left[\frac{n}{2}\right]} b_{k, n} \frac{\cos 2 k \theta}{n-2 k+\mu} \text { and } \lim _{n \rightarrow \infty} \frac{n}{\left(\begin{array}{c}
n+d-1 \\
n
\end{array}\right)} \sum_{k=0}^{\left[\frac{n}{2}\right]} b_{k, n} \frac{\sin 2 k \theta}{n-2 k+\mu}
$$

are equal to zero. Again, we only need to deal with $d=2$ and $d=3$. For $d=3$, the two limits become

$$
\lim _{n \rightarrow \infty} \frac{n}{\left(\begin{array}{c}
n+2 \\
n
\end{array}\right)} \sum_{k=0}^{\left[\frac{n}{2}\right]} \cos 2 k \theta \quad \text { and } \quad \lim _{n \rightarrow \infty} \frac{n}{\left(\begin{array}{c}
n+2 \\
n
\end{array}\right)} \sum_{k=0}^{\left[\frac{n}{2}\right]} \sin 2 k \theta .
$$

That the limits are zero for $0<\theta<\pi$ follows readily from the well-known identities

$$
\frac{1}{2}+\sum_{k=1}^{m} \cos k \theta=\frac{\sin \left(m+\frac{1}{2}\right) \theta}{2 \sin \frac{\theta}{2}}, \quad \sum_{k=1}^{m} \sin k \theta=\frac{\cos \frac{\theta}{2}-\cos \left(m+\frac{1}{2}\right) \theta}{2 \sin \frac{\theta}{2}},
$$

which shows that the two sums are bounded independent of $m$ for $0<\theta<\pi$. The case $d=2$ is a little more complicated. Following the reduction in the proof for the 
case (A), it amounts to proving that

$$
\lim _{m \rightarrow \infty} \sum_{k=0}^{m-1} \frac{\cos 2 k \theta}{\sqrt{m^{2}-k^{2}}}=0 \quad \text { and } \quad \lim _{m \rightarrow \infty} \sum_{k=0}^{m-1} \frac{\sin 2 k \theta}{\sqrt{m^{2}-k^{2}}}=0, \quad 0<\theta<\pi .
$$

Using summation by parts, we see that the first sum becomes

$$
\begin{gathered}
\sum_{k=0}^{m-1} \frac{\cos 2 k \theta}{\sqrt{m^{2}-k^{2}}}=\sum_{k=0}^{m-2}\left(\frac{1}{\sqrt{m^{2}-k^{2}}}-\frac{1}{\sqrt{m^{2}-(k+1)^{2}}}\right) \sum_{j=0}^{k} \cos 2 j \theta \\
+\frac{1}{\sqrt{m^{2}-(m-1)^{2}}} \sum_{j=0}^{m-1} \cos 2 j \theta .
\end{gathered}
$$

Therefore, for $0<\theta<\pi$, we obtain from the boundedness of the cosine sums as shown by (3.15) that

$$
\begin{aligned}
\sum_{k=0}^{m-1} \frac{\cos 2 k \theta}{\sqrt{m^{2}-k^{2}}}=\mathcal{O} & (1)\left[\sum_{k=0}^{m-2}\left(\frac{1}{\sqrt{m^{2}-(k+1)^{2}}}-\frac{1}{\sqrt{m^{2}-k^{2}}}\right)\right. \\
& \left.+\frac{1}{\sqrt{m^{2}-(m-1)^{2}}}\right]=\mathcal{O}\left(\frac{1}{\sqrt{m}}\right)
\end{aligned}
$$

which verifies the sum of cosines, the sum of sines is verified similarly. That completes the proof of $(\mathrm{B})$.

To prove (C), we note that by (3.12), the left-hand side of $(C)$ is equal to

$$
I_{n}:=\mathcal{O}(1) \frac{n}{\left(\begin{array}{c}
n+d-1 \\
n
\end{array}\right)} \sum_{k=0}^{\left[\frac{n}{2}\right]} \frac{\Gamma\left(k+\frac{d-1}{2}\right) \Gamma\left(n-k+\mu+\frac{d-1}{2}\right)}{\Gamma(k+1) \Gamma(n-k+\mu+1)} \frac{1}{(n-2 k+\mu)^{2}} .
$$

Since $n-k \sim n$ for $1 \leq-k \leq\left[\frac{n}{2}\right]$, we have by (2.12) that

$$
I_{n}=\mathcal{O}(1) \frac{1}{n^{\frac{d-1}{2}}} \sum_{k=0}^{\left[\frac{n}{2}\right]} \frac{k^{\frac{d-3}{2}}}{(n-2 k+\mu)^{2}}
$$

To show that $I_{n}$ goes to zero as $N \rightarrow \infty$, we split the summation into two parts,

$$
I_{n}=\mathcal{O}(1) \frac{1}{n^{\frac{d-1}{2}}}\left(\sum_{k=0}^{\left[\frac{n}{2}\right]-\sqrt{\left[\frac{n}{2}\right]}}+\sum_{k=\left[\frac{n}{2}\right]-\sqrt{\left[\frac{n}{2}\right]}}^{\left[\frac{n}{2}\right]}\right) \frac{k^{\frac{d-3}{2}}}{(n-2 k+\mu)^{2}} .
$$

For the first sum, we use the fact that $n-2 k+\mu \geq c \sqrt{n}$, and for the second one, we use the fact that $2 k \sim n$; it follows readily that

$$
I_{n}=\mathcal{O}(1) \frac{1}{n^{\frac{d-1}{2}}}\left[\frac{n^{\frac{d-1}{2}}}{n}+n^{\frac{d-3}{2}} n^{\frac{1}{2}}\right]=\mathcal{O}(1) \frac{1}{\sqrt{n}} \rightarrow 0 .
$$

This proves (C). Together with (A) and (B), we have established (3.6) for $0<|\mathbf{x}|<1$.

The proof of (3.6) for $|\mathbf{x}|=1$ follows easily from (2.9), (2.12) and the fact that

$$
\left|\mathbb{P}_{n}^{(\mu)}\right|^{2}(1)=\frac{n+\mu+\frac{d-1}{2}}{\mu+\frac{d-1}{2}} C_{n}^{\left(\mu+\frac{d-1}{2}\right)}(1) .
$$

Finally, to prove (3.7), we set $\mathbf{x}=\mathbf{y}=0$ in (2.5) to obtain

$$
\left|\mathbb{P}_{n}^{(\mu)}\right|^{2}(0)=\frac{n+\mu+\frac{d-1}{2}}{\mu+\frac{d-1}{2}} \int_{0}^{\pi} C_{n}^{\left(\mu+\frac{d-1}{2}\right)}(\cos \psi)(\sin \psi)^{2 \mu-1} d \psi / \int_{0}^{\pi}(\sin \psi)^{2 \mu-1} d \psi
$$


From (2.9), it follows readily that $\left|\mathbb{P}_{n}^{(\mu)}\right|^{2}(0)=0$ for odd $n$. For even integer $n$, we use the formula $(2.8)$ to connect $C_{n}^{\left(\mu+\frac{d-1}{2}\right)}$ and $C_{n}^{\left(\mu-\frac{1}{2}\right)}$; using the orthogonality, the coefficient of $C_{0}^{\left(\mu-\frac{1}{2}\right)}$ in the connection formula gives

$$
\left|\mathbb{P}_{n}^{(\mu)}\right|^{2}(0)=\frac{n+\mu+\frac{d-1}{2}}{\mu+\frac{d-1}{2}} \frac{\Gamma\left(\mu+\frac{1}{2}\right)}{\Gamma\left(\mu+\frac{d-1}{2}\right) \Gamma\left(\frac{d}{2}\right)} \frac{\Gamma\left(\frac{n+d}{2}\right) \Gamma\left(\frac{n}{2}+\mu+\frac{d-1}{2}\right)}{\Gamma\left(\frac{n}{2}+1\right) \Gamma\left(\frac{n}{2}+\mu+\frac{1}{2}\right)} .
$$

By (2.12) and (3.13), it follows readily that

$$
\frac{1}{\left(\begin{array}{c}
n+d-1 \\
n
\end{array}\right)}\left|\mathbb{P}_{n}^{(\mu)}\right|^{2}(0)=2 \frac{\Gamma\left(\mu+\frac{1}{2}\right) \Gamma\left(\frac{d+1}{2}\right)}{\Gamma\left(\mu+\frac{d+1}{2}\right) \Gamma\left(\frac{1}{2}\right)}\left(1+\mathcal{O}\left(\frac{1}{n}\right)\right)=2 \frac{w_{0}}{w_{\mu}}\left(1+\mathcal{O}\left(\frac{1}{n}\right)\right) .
$$

Thus, we have completed the proof of the theorem.

\section{Asymptotics of the Christoffel functions}

First, we derive the asymptotics of the Christoffel functions with respect to $W_{\mu}$, which we denote by $\Lambda_{n, d}^{(\mu)}$.

Theorem 4.1. For $W_{\mu, d}, \mu \geq 0, d \geq 2$,

$$
\lim _{n \rightarrow \infty}\left(\begin{array}{c}
n+d \\
n
\end{array}\right) \Lambda_{n, d}^{(\mu)}(\mathbf{x})=\frac{W_{\mu}(\mathbf{x})}{W_{0}(\mathbf{x})}, \quad|\mathbf{x}|<1
$$

and

$$
\lim _{n \rightarrow \infty}\left(\begin{array}{c}
n+d \\
n
\end{array}\right) \Lambda_{n, d}^{(\mu)}(1)= \begin{cases}0, & \text { if } \mu>0 \\
\frac{1}{2}, & \text { if } \mu=0\end{cases}
$$

Proof. From (3.10), it follows readily that

$$
\lim _{n \rightarrow \infty} \frac{\mathbf{K}_{n, d}^{(\mu)}(|\mathbf{x}|)}{\left(\begin{array}{c}
n+d \\
n
\end{array}\right)}=\lim _{n \rightarrow \infty} \frac{\left|\mathbb{P}_{n, d}^{(\mu)}\right|^{2}(|\mathbf{x}|)}{\left(\begin{array}{c}
n+d \\
n
\end{array}\right)-\left(\begin{array}{c}
n-1+d \\
n-1
\end{array}\right)}=\lim _{n \rightarrow \infty} \frac{\left|\mathbb{P}_{n, d}^{(\mu)}\right|^{2}(|\mathbf{x}|)}{\left(\begin{array}{c}
n+d-1 \\
n
\end{array}\right)}
$$

Thus, except for the case $\mu=0$ and $d=2$, the desired limit for $0<|\mathbf{x}| \leq 1$ follows from that of Theorems 3.2 and 3.3. So does the limit at $|\mathbf{x}|=1$ for the case $\mu=0$ and $d=2$. For $\mu>0,\left|\mathbb{P}_{n, d}^{(\mu)}\right|^{2}(0)=0$ for odd integers $n$, which allows us to write

$$
\lim _{n \rightarrow \infty} \frac{\mathbf{K}_{n, d}^{(\mu)}(0)}{\left(\begin{array}{c}
n+d \\
n
\end{array}\right)}=\lim _{n \rightarrow \infty} \frac{\left|\mathbb{P}_{2 n, d}^{(\mu)}\right|^{2}(0)}{\left(\begin{array}{c}
2 n+d \\
2 n
\end{array}\right)-\left(\begin{array}{c}
2 n-2+d \\
2 n-2
\end{array}\right)}=\lim _{n \rightarrow \infty} \frac{\left|\mathbb{P}_{2 n, d}^{(\mu)}\right|^{2}(|\mathbf{x}|)}{2\left(\begin{array}{c}
2 n+d-1 \\
2 n
\end{array}\right)}=\frac{W_{0}(\mathbf{x})}{W_{\mu}(\mathbf{x})}
$$

where the last step follows from (3.7). The only remaining case is the limit for $0<$ $|\mathbf{x}|<1$ in the case of $d=2$ and $\mu=0$, for which the limit of $\left|\mathbb{P}_{n, 2}^{(\mu)}\right|^{2}$ does not exist. However, setting $\mathbf{x}=\mathbf{y}$ in (2.6) gives

$$
\mathbf{K}_{n, d}^{(0)}=\frac{1}{2}\left[C_{n}^{\left(\frac{d+1}{2}\right)}(1)+C_{n-1}^{\left(\frac{d+1}{2}\right)}(1)+C_{n}^{\left(\frac{d+1}{2}\right)}\left(2|\mathbf{x}|^{2}-1\right)+C_{n-1}^{\left(\frac{d+1}{2}\right)}\left(2|\mathbf{x}|^{2}-1\right)\right],
$$

from which the desired limit follows easily from (2.9) and (2.11).

As we mentioned in the introduction, the limit (4.1) has been proved by Bos in [3] using a different method.

Next, we derive an upper bound for the Christoffel functions with respect to a general class of radial weight functions. For a weight function $W$, we denote the 
Christoffel functions associated with it by $\Lambda_{n}(W)$. The result depends on the following compact formula for $\mathbf{K}_{n}^{(0)}(\mathbf{x}, \mathbf{y})([12])$ :

$$
\begin{aligned}
& \mathbf{K}_{n}^{(0)}(\mathbf{x}, \mathbf{y})= \\
& \quad \frac{1}{2}\left[C_{n}^{\left(\frac{d+1}{2}\right)}\left(\mathbf{x} \cdot \mathbf{y}+\sqrt{1-|\mathbf{x}|^{2}} \sqrt{1-|\mathbf{y}|^{2}}\right)+C_{n-1}^{\left(\frac{d+1}{2}\right)}\left(\mathbf{x} \cdot \mathbf{y}+\sqrt{1-|\mathbf{x}|^{2}} \sqrt{1-|\mathbf{y}|^{2}}\right)\right] \\
& \quad+\frac{1}{2}\left[C_{n}^{\left(\frac{d+1}{2}\right)}\left(\mathbf{x} \cdot \mathbf{y}-\sqrt{1-|\mathbf{x}|^{2}} \sqrt{1-|\mathbf{y}|^{2}}\right)+C_{n-1}^{\left(\frac{d+1}{2}\right)}\left(\mathbf{x} \cdot \mathbf{y}-\sqrt{1-|\mathbf{x}|^{2}} \sqrt{1-|\mathbf{y}|^{2}}\right)\right],
\end{aligned}
$$

which is derived from (2.6) using (3.3). For a radial function $f$ on $\mathbb{R}^{d}$, we denote by $\tilde{f}: \mathbb{R}_{+} \rightarrow \mathbb{R}$ the function such that $f(\mathbf{x})=\tilde{f}(|\mathbf{x}|)$.

Theorem 4.2. Let $W$ be a nonnegative radial function on $\mathbb{R}^{d}$, such that $W / W_{0}$ is continuous in the interior of $B^{d}$ and is bounded on $B^{d}$. Then,

$$
\limsup _{n \rightarrow \infty}\left(\begin{array}{c}
n+d \\
n
\end{array}\right) \Lambda_{n}(W ; \mathbf{x}) \leq \frac{W(\mathbf{x})}{W_{0}(\mathbf{x})}, \quad|\mathbf{x}|<1 .
$$

Proof. Because of (1.4), we have

$$
\left(\begin{array}{c}
n+d \\
n
\end{array}\right) \Lambda_{n}(W ; \mathbf{x}) \leq\left(\begin{array}{c}
n+d \\
n
\end{array}\right) \frac{1}{\left[\mathbf{K}_{n}^{(0)}(\mathbf{x}, \mathbf{x})\right]^{2}} \int_{[-1,1]^{d}}\left[\mathbf{K}_{n}^{(0)}(\mathbf{x}, \mathbf{y})\right]^{2} W(\mathbf{y}) d \mathbf{y} .
$$

By (4.1) with $\mu=0$, it follows readily that the desired result is the consequence of the limit

$$
\lim _{n \rightarrow \infty} \frac{1}{\mathbf{K}_{n}^{(0)}(\mathbf{x}, \mathbf{x})} \int_{[-1,1]^{d}}\left[\mathbf{K}_{n}^{(0)}(\mathbf{x}, \mathbf{y})\right]^{2} W(\mathbf{y}) d \mathbf{y}=\frac{W(\mathbf{x})}{W_{0}(\mathbf{x})}, \quad|\mathbf{x}|<1,
$$

which is a corollary of the theorem below.

Theorem 4.3. Let $f$ be a radial function on $\mathbb{R}^{d}$ such that $f$ is continuous in the interior of $B^{d}$ and bounded on $B^{d}$. Then,

$$
\lim _{n \rightarrow \infty} \frac{1}{\mathbf{K}_{n}^{(0)}(\mathbf{x}, \mathbf{x})} \int_{[-1,1]^{d}}\left[\mathbf{K}_{n}^{(0)}(\mathbf{x}, \mathbf{y})\right]^{2} f(\mathbf{y}) W_{0}(\mathbf{y}) d \mathbf{y}=f(\mathbf{x}), \quad|\mathbf{x}|<1 .
$$

Proof. By the definition of $\mathbf{K}_{n}(\cdot, \cdot)$ in (1.2), it follows readily from the orthogonality that

$$
\int_{[-1,1]^{d}}\left[\mathbf{K}_{n}^{(0)}(\mathbf{x}, \mathbf{y})\right]^{2} W_{0}(\mathbf{y}) d \mathbf{y}=\mathbf{K}_{n}^{(0)}(\mathbf{x}, \mathbf{x}) .
$$

Therefore, it follows readily that

$$
\begin{aligned}
I_{n}(\mathbf{x}) & :=\left|\frac{1}{\mathbf{K}_{n}^{(0)}(\mathbf{x}, \mathbf{x})} \int_{[-1,1]^{d}}\left[\mathbf{K}_{n}^{(0)}(\mathbf{x}, \mathbf{y})\right]^{2} f(\mathbf{y}) W_{0}(\mathbf{y}) d \mathbf{y}-f(\mathbf{x})\right| \\
& \leq \frac{1}{\mathbf{K}_{n}^{(0)}(\mathbf{x}, \mathbf{x})} \int_{[-1,1]^{d}}\left[\mathbf{K}_{n}^{(0)}(\mathbf{x}, \mathbf{y})\right]^{2}|f(\mathbf{y})-f(\mathbf{x})| W_{0}(\mathbf{y}) d \mathbf{y}
\end{aligned}
$$

Since $f$ is a radial function on $B^{d}$, the function $\tilde{f}$ is defined on $[0,1]$; moreover, by assumption, $\tilde{f}$ is continuous on [0,1). For the following, fix $\mathbf{x}$ inside $B^{d}$. Let $\varepsilon>0$ be chosen such that

$$
B_{\varepsilon}(\mathbf{x}):=\left\{\mathbf{y} \in B^{d}:|| \mathbf{y}|-| \mathbf{x}||<\varepsilon\right\} \subset B^{d} .
$$


Let $\omega(\tilde{f})$ be the modulus of continuity of $\tilde{f}$, and let $\|f\|_{\infty}$ denote the uniform norm of $f$ on $B^{d}$. We have

$$
\begin{aligned}
I_{n}(\mathbf{x}) \leq & \omega(\tilde{f}, \varepsilon) \frac{1}{\mathbf{K}_{n}^{(0)}(\mathbf{x}, \mathbf{x})} \int_{B_{\varepsilon}(\mathbf{x})}\left[\mathbf{K}_{n}^{(0)}(\mathbf{x}, \mathbf{y})\right]^{2} W_{0}(\mathbf{y}) d \mathbf{y} \\
& +\frac{1}{\mathbf{K}_{n}^{(0)}(\mathbf{x}, \mathbf{x})} \int_{B^{d} \backslash B_{\varepsilon}(\mathbf{x})}\left[\mathbf{K}_{n}^{(0)}(\mathbf{x}, \mathbf{y})\right]^{2}|f(\mathbf{x})-f(\mathbf{y})| W_{0}(\mathbf{y}) d \mathbf{y} \\
\leq & \omega(\tilde{f}, \varepsilon)+2\|f\|_{\infty} \frac{1}{\mathbf{K}_{n}^{(0)}(\mathbf{x}, \mathbf{x})} \int_{B^{d} \backslash B_{\varepsilon}(\mathbf{x})}\left[\mathbf{K}_{n}^{(0)}(\mathbf{x}, \mathbf{y})\right]^{2} W_{0}(\mathbf{y}) d \mathbf{y}
\end{aligned}
$$

By the definition of $\mathbf{K}_{n}^{(0)}$ in (4.3) and the limit (4.1) with $\mu=0$, it suffices to prove that

$$
\lim _{n \rightarrow \infty} \frac{1}{\left(\begin{array}{c}
n+d \\
n
\end{array}\right)} \int_{B^{d} \backslash B_{\varepsilon}(\mathbf{x})}\left[C_{n}^{\left(\frac{d+1}{2}\right)}\left(\mathbf{x} \cdot \mathbf{y} \pm \sqrt{1-|\mathbf{x}|^{2}} \sqrt{1-|\mathbf{y}|^{2}}\right)\right]^{2} W_{0}(\mathbf{y}) d \mathbf{y}=0 .
$$

Moreover, it follows from a change of variable $\mathbf{y} \mapsto-\mathbf{z}$ that we only need to deal with the case of $\mathbf{x} \cdot \mathbf{y}+\sqrt{1-|\mathbf{x}|^{2}} \sqrt{1-|\mathbf{y}|^{2}}$. We use the asymptotics of ultraspherical polynomials in (3.14), which imply, by (2.9), that

$$
\frac{1}{\left(\begin{array}{c}
n+d \\
n
\end{array}\right)}\left[C_{n}^{\left(\frac{d+1}{2}\right)}(t)\right]^{2}=\frac{\Gamma\left(\frac{1}{2}\right) \Gamma\left(\frac{d+2}{2}\right)}{\Gamma\left(\frac{d+1}{2}\right)} \frac{2}{\pi} \frac{1}{n+\frac{d+1}{2}}\left[\frac{1}{\left(1-t^{2}\right)^{\frac{d+1}{2}}} \cos ^{2}(N \theta+\gamma)+\mathcal{O}\left(n^{-1}\right)\right]
$$

where $N=n+(d+1) / 2, \gamma=(d+1) \pi / 4, t=\cos \theta$. Using the identities

$$
2 \mathbf{x} \cdot \mathbf{y}=|\mathbf{x}|^{2}+|\mathbf{y}|^{2}-|\mathbf{x}-\mathbf{y}|^{2} \quad \text { and } \quad 2 \mathbf{x} \cdot \mathbf{y}=|\mathbf{x}+\mathbf{y}|^{2}-|\mathbf{x}|^{2}-|\mathbf{y}|^{2}
$$

it follows that for $\mathbf{y} \in B^{d} \backslash B_{\varepsilon}(\mathbf{x})$,

$$
1-\mathbf{x} \cdot \mathbf{y}-\sqrt{1-|\mathbf{x}|^{2}} \sqrt{1-|\mathbf{y}|^{2}}=\frac{1}{2}\left(\sqrt{1-|\mathbf{x}|^{2}}-\sqrt{1-|\mathbf{y}|^{2}}\right)^{2}+\frac{1}{2}|\mathbf{x}-\mathbf{y}|^{2} \geq \frac{\varepsilon}{2}
$$

and

$$
1+\mathbf{x} \cdot \mathbf{y}+\sqrt{1-|\mathbf{x}|^{2}} \sqrt{1-|\mathbf{y}|^{2}}=\frac{1}{2}\left(\sqrt{1-|\mathbf{x}|^{2}}+\sqrt{1-|\mathbf{y}|^{2}}\right)^{2}+\frac{1}{2}|\mathbf{x}+\mathbf{y}|^{2} \geq \frac{\varepsilon}{2} .
$$

Let $t=\mathbf{x} \cdot \mathbf{y}+\sqrt{1-|\mathbf{x}|^{2}} \sqrt{1-|\mathbf{y}|^{2}}$. Then, the above inequalities imply that

$$
1-t^{2}=(1-t)(1+t) \geq \frac{\varepsilon^{2}}{4}, \quad \mathbf{y} \in B^{d} \backslash B_{\varepsilon}(\mathbf{x})
$$

Hence, it follows that

$$
\begin{aligned}
& \frac{1}{\left(\begin{array}{c}
n+d \\
n
\end{array}\right)}\left[C_{n}^{\left(\frac{d+1}{2}\right)}\left(\mathbf{x} \cdot \mathbf{y}+\sqrt{1-|\mathbf{x}|^{2}} \sqrt{1-|\mathbf{y}|^{2}}\right)\right]^{2} \\
& \quad=\mathcal{O}(1) \frac{1}{n \varepsilon^{d+1}}+\mathcal{O}\left(\frac{1}{n^{2}}\right), \quad \mathbf{y} \in B^{d} \backslash B_{\varepsilon}(\mathbf{x})
\end{aligned}
$$

Therefore, we have that

$$
\begin{aligned}
& \frac{1}{\left(\begin{array}{c}
n+d \\
n
\end{array}\right)} \int_{B^{d} \backslash B_{\varepsilon}(\mathbf{x})}\left[C_{n}^{\left(\frac{d+1}{2}\right)}\left(\mathbf{x} \cdot \mathbf{y} \pm \sqrt{1-|\mathbf{x}|^{2}} \sqrt{1-|\mathbf{y}|^{2}}\right)\right]^{2} W_{0}(\mathbf{y}) d \mathbf{y} \\
& \quad=\mathcal{O}(1) \frac{1}{n \varepsilon^{d+1}}+\mathcal{O}\left(\frac{1}{n^{2}}\right)
\end{aligned}
$$

which clearly goes to zero as $n \rightarrow \infty$. This completes the proof. 
For orthogonal polynomials in one variable, the limit process in the theorem has been studied for general weight functions (cf. [7]). We remark that, under more restricted conditions on $W / W_{0}$, we can use an approach of Freud as in [11, Theorem 4.3.1] to prove

$$
c \frac{W(\mathbf{x})}{W_{0}(\mathbf{x})} \leq \liminf _{n \rightarrow \infty}\left(\begin{array}{c}
n+d \\
n
\end{array}\right) \Lambda_{n}(W ; x), \quad|\mathbf{x}|<1,
$$

with a constant $c$ less than 1 (say, $c=2^{-d}$ ). However, it is almost certain that such a relation should be true with $c=1$ and without additional restrictions, which means that the limit as in Theorem 4.1 holds. For this reason, we do not include a proof of the weaker result (4.4).

\section{A recurrence relation}

From the formulae (3.8) and (2.7), we see that the polynomial $\left|\mathbb{P}_{n}^{(\mu)}\right|^{2}(r)$ acts like a square of an ultraspherical polynomial. In this section, we derive an interesting relation among $\left|\mathbb{P}_{n}^{(\mu)}\right|^{2}(r)$ of different $n$ and $\mu$ 's.

Theorem 5.1. For $\mu>0$ and $n \geq 0$,

$$
\begin{aligned}
& \left(1-|\mathbf{x}|^{2}\right)\left(1-|\mathbf{y}|^{2}\right)\left[\mathbb{P}_{n}^{(\mu+1)}(\mathbf{x})\right]^{T} \mathbb{P}_{n}^{(\mu+1)}(\mathbf{y}) \\
& =\frac{\mu+\frac{1}{2}}{\mu+\frac{d+1}{2}}\left[\frac{n+2}{2\left(n+\mu+\frac{d+3}{2}\right)}\left[\mathbb{P}_{n+2}^{(\mu)}(\mathbf{x})\right]^{T} \mathbb{P}_{n+2}^{(\mu)}(\mathbf{y})\right. \\
& \left.\quad+\frac{n+2 \mu+d-1}{2\left(n+\mu+\frac{d-1}{2}\right)}\left[\mathbb{P}_{n}^{(\mu)}(\mathbf{x})\right]^{T} \mathbb{P}_{n}^{(\mu)}(\mathbf{y})-\mathbf{x} \cdot \mathbf{y}\left[\mathbb{P}_{n+1}^{(\mu)}(\mathbf{x})\right]^{T} \mathbb{P}_{n+1}^{(\mu)}(\mathbf{y})\right]
\end{aligned}
$$

In particular,

$$
\begin{aligned}
\left(1-|\mathbf{x}|^{2}\right)^{2}\left|\mathbb{P}_{n}^{(\mu+1)}\right|^{2}(|\mathbf{x}|)= & \frac{\mu+\frac{1}{2}}{\mu}+\frac{n+2}{2}\left[\frac{d+1}{2\left(n+\mu+\frac{d+3}{2}\right)}\left|\mathbb{P}_{n+2}^{(\mu)}\right|^{2}(|\mathbf{x}|)\right. \\
& \left.+\frac{n+2 \mu+d-1}{2\left(n+\mu+\frac{d-1}{2}\right)}\left|\mathbb{P}_{n}^{(\mu)}\right|^{2}(|\mathbf{x}|)-|\mathbf{x}|^{2} \mathbb{P}_{n+1}^{(\mu)}{ }^{T}(|\mathbf{x}|)\right] .
\end{aligned}
$$

Proof. Let $A=\mathbf{x} \cdot \mathbf{y}$ and $B=\sqrt{1-|\mathbf{x}|^{2}} \sqrt{1-|\mathbf{y}|^{2}}$. We start with the formula

$$
\frac{d}{d \psi} C_{n+1}^{(\lambda)}(A+B \cos \psi)=-2 \lambda B \sin \psi C_{n}^{(\lambda+1)}(A+B \cos \psi)
$$

which follows from the fact that $\left[C_{n+1}^{(\lambda)}(x)\right]^{\prime}=2 \lambda C_{n}^{(\lambda+1)}(x)$ (cf. $[8$, p. 81, (4.7.14)]) and a simple change of variable. Using this formula and integration by parts, we have

$$
\begin{aligned}
\int_{0}^{\pi} C_{n}^{\left(\mu+\frac{d+1}{2}\right)}(A+B \cos \psi)(\sin \psi)^{2 \mu+1} d \psi \\
\quad=\frac{-1}{(2 \mu+d-1) B} \int_{0}^{\pi} \frac{d}{d \psi} C_{n+1}^{\left(\mu+\frac{d-1}{2}\right)}(A+B \cos \psi)(\sin \psi)^{2 \mu} d \psi \\
\quad=\frac{2 \mu}{(2 \mu+d-1) B} \int_{0}^{\pi} C_{n+1}^{\left(\mu+\frac{d-1}{2}\right)}(A+B \cos \psi) \cos \psi(\sin \psi)^{2 \mu-1} d \psi
\end{aligned}
$$

Writing $\cos \psi$ as

$$
\cos \psi=\frac{1}{B}(A+B \cos \psi)-\frac{A}{B}
$$


and applying the three-term relation for the ultraspherical polynomials ([8, p. 81 , 4.7.18]), we conclude that

$$
\begin{aligned}
\int_{0}^{\pi} C_{n}^{\left(\mu+\frac{d+1}{2}\right)}(A+B \cos \psi)(\sin \psi)^{2 \mu+1} d \psi \\
=\frac{2 \mu}{(2 \mu+d-1) B}\left[\frac{n+2}{(2 n+2 \mu+d+1) B} \int_{0}^{\pi} C_{n+2}^{\left(\mu+\frac{d-1}{2}\right)}(A+B \cos \psi)(\sin \psi)^{2 \mu-1} d \psi\right. \\
\quad+\frac{n+2 \mu+d-1}{(2 n+2 \mu+d+1) B} \int_{0}^{\pi} C_{n}^{\left(\mu+\frac{d-1}{2}\right)}(A+B \cos \psi)(\sin \psi)^{2 \mu-1} d \psi \\
\left.\quad-\frac{A}{B} \int_{0}^{\pi} C_{n+1}^{\left(\mu+\frac{d-1}{2}\right)}(A+B \cos \psi)(\sin \psi)^{2 \mu-1} d \psi\right] .
\end{aligned}
$$

Using the formula (2.5) and the fact that

$$
c_{\mu+1}^{-1}=\int_{0}^{\pi}(\sin \psi)^{2 \mu+1} d \psi=\frac{2 \mu}{2 \mu+1} c_{\mu}^{-1},
$$

the desired formula follows.

For the case $\mu=0$, the identities in Theorem 5.1 take a different form.

Theorem 5.2. For $n \geq 0$,

$$
\begin{gathered}
\frac{1}{2}\left[\mathbb{P}_{n+1}^{(0)}(\mathbf{x})\right]^{T} \mathbb{P}_{n+1}^{(0)}(\mathbf{y}) \pm \frac{d+1}{2} \sqrt{1-|\mathbf{x}|^{2}} \sqrt{1-|\mathbf{y}|^{2}}\left[\mathbb{P}_{n}^{(1)}(\mathbf{x})\right]^{T} \mathbb{P}_{n}^{(1)}(\mathbf{y}) \\
=\frac{n+\frac{d+1}{2}}{d-1} C_{n+1}^{\frac{d-1}{2}}\left(\mathbf{x} \cdot \mathbf{y} \mp \sqrt{1-|\mathbf{x}|^{2}} \sqrt{1-|\mathbf{y}|^{2}}\right) .
\end{gathered}
$$

In particular,

$$
\frac{1}{2}\left|\mathbb{P}_{n+1}^{(0)}\right|^{2}(|\mathbf{x}|)+\frac{d+1}{2}\left(1-|\mathbf{x}|^{2}\right)\left|\mathbb{P}_{n}^{(1)}\right|^{2}(|\mathbf{x}|)=\frac{n+\frac{d-1}{2}}{n+1} C_{n+1}^{\left(\frac{d-1}{2}\right)}(1) .
$$

Proof. The proof is similar to that of the previous theorem, but the special value of the parameter allows us to integrate directly instead of doing integration by parts. It follows that

$$
\begin{aligned}
\sqrt{1-|\mathbf{x}|^{2}} \sqrt{1-|\mathbf{y}|^{2}}\left[\mathbb{P}_{n}^{(1)}(\mathbf{x})\right]^{T} \mathbb{P}_{n}^{(1)}(\mathbf{y}) \\
=\frac{n+\frac{d+1}{2}}{(d+1) d}\left[C_{n+1}^{\left(\frac{d-1}{2}\right)}\left(\mathbf{x} \cdot \mathbf{y}+\sqrt{1-|\mathbf{x}|^{2}} \sqrt{1-|\mathbf{y}|^{2}}\right)\right. \\
\left.\quad-C_{n+1}^{\left(\frac{d-1}{2}\right)}\left(\mathbf{x} \cdot \mathbf{y}-\sqrt{1-|\mathbf{x}|^{2}} \sqrt{1-|\mathbf{y}|^{2}}\right)\right]
\end{aligned}
$$

Together with (2.6), the above formula implies the desired results.

The formula (5.2) and (5.4) allows us to deduce the asympotic formula for $\left|\mathbb{P}_{n+1}^{(\mu+1)}\right|^{2}(r)$ from that of $\left|\mathbb{P}_{n+1}^{(\mu)}\right|^{2}(r)$. Therefore, in the proof of the limit (3.6) in Theorem 3.3, we could have restricted the parameter $\mu$ to $0 \leq \mu<1$. In particular, since the limit relation is easily established in the case of $\mu=0$, it follows that the limit (3.6) for the integer values of $\mu$ can be established effortless, by the use of (5.2) and (5.4).

We denote by $\tilde{C}_{n}^{(\mu)}$ the orthonormal polynomials with respect to $W_{\mu, 1}$, which is a constant multiple of $C_{n}^{(\mu)}$. Then, in particular, for $d=1$ and $n \geq 0$,

$$
\mathbb{P}_{n}^{(1)}(x)=\tilde{C}_{n}^{(1)}(x)=\frac{\sin (n+1) \theta}{\sin \theta}, \quad \mathbb{P}_{n+1}^{(0)}(x)=\tilde{C}_{n+1}^{(0)}(x)=\cos (n+1) \theta, \quad x=\cos \theta .
$$


Taking the limit (2.4) in the right-hand side of (5.3), we obtain the elementary trigonometric formula

$$
\cos (n+1) \theta \cos (n+1) \phi \pm \sin (n+1) \theta \sin (n+1) \phi=\cos (n+1)(\theta \mp \phi) .
$$

The formula (5.4) for $d=1$ becomes

$$
\cos ^{2}(n+1) \theta+\sin ^{2}(n+1) \theta=1 .
$$

We also can sum up the identities in Theorem 5.1 and Theorem 5.2 to obtain identities involving $\mathbf{K}_{n}^{(\mu)}$. Instead of presenting the formula for general $\mu$, we state only the following special case.

Theorem 5.3. For $n \geq 0$,

In particular,

$$
\begin{array}{r}
\frac{1}{d+1} \mathbf{K}_{n+1}^{(0)}(\mathbf{x}, \mathbf{y})+\sqrt{1-|\mathbf{x}|^{2}} \sqrt{1-|\mathbf{y}|^{2}} \mathbf{K}_{n}^{(1)}(\mathbf{x}, \mathbf{y}) \\
=\frac{1}{d+1}\left[C_{n+1}^{\left(\frac{d+1}{2}\right)}\left(\mathbf{x} \cdot \mathbf{y}+\sqrt{1-|\mathbf{x}|^{2}} \sqrt{1-|\mathbf{y}|^{2}}\right)\right. \\
\left.+C_{n}^{\left(\frac{d+1}{2}\right)}\left(\mathbf{x} \cdot \mathbf{y}+\sqrt{1-|\mathbf{x}|^{2}} \sqrt{1-|\mathbf{y}|^{2}}\right)\right]
\end{array}
$$

$$
\frac{1}{d+1} \mathbf{K}_{n+1}^{(0)}(\mathbf{x}, \mathbf{x})+\left(1-|\mathbf{x}|^{2}\right) \mathbf{K}_{n}^{(1)}(\mathbf{x}, \mathbf{y})=\frac{1}{d+1}\left[C_{n+1}^{\left(\frac{d+1}{2}\right)}(1)+C_{n}^{\left(\frac{d+1}{2}\right)}(1)\right] .
$$

We remark that the formula (5.6) has appeared in [3, Lemma 1], where the proof is rather complicated but nevertheless interesting. The constants in the formula in [3] appear to be different from ours, which is due to a different normalization of the weight function.

Acknowledgment. This work is supported by the National Science Foundation under Grant DMS-9302721 and 9500532.

\section{References}

1. M. Abramowitz and I. Stegun, Handbook of Mathematical Functions, 9th print., Dover Publ., New York, 1970.

2. R. Askey, Orthogonal Polynomials and Special Functions, Reg. Conf. Series in Appl. Math. 21, SIAM, Philadelphia, 1975.

3. L. Bos, Asymptotics for the Christoffel function for Jacobi like weights on a ball in $\mathbb{R}^{m}$, New Zealand J. Math., 23 (1994), 99-109.

4. T. J. Bromwich, Introduction to the Theory of Infinite Series, 2nd ed., MacMillan, London, 1926.

5. A. Erdélyi, et al, Higher Transcendental Functions, McGraw-Hill, New York, 1953.

6. T. Koornwinder, Two-variable analogues of the classical orthogonal polynomials, in Theory and Applications of Special Functions, (R. A. Askey ed.), Academic Press, 1975, pp. 435-495.

7. P. Nevai, Géza Freud, orthogonal polynomials and Christoffel functions. A case study, J. Approx. Theory, 48 (1986), 3-167.

8. G. Szegö, Orthogonal Polynomials, Amer. Math. Soc. Colloq. Publ. Vol.23, Providence, 4th edition, 1975.

9. Y. Xu, On multivariate orthogonal polynomials, SIAM J. Math. Anal. 24 (1993), 783-794.

10. C_ Common Zeros of Polynomials in Several Variables and Higher Dimensional Quadrature, Pitman Research Notes in Mathematics Series, Longman, Essex, 1994. 
11. Christoffel functions and Fourier Series for multivariate orthogonal polynomials, J. Approx. Theory, 82 (1995), 205-239.

12. Summability of Fourier orthogonal series for Jacobi weight on a ball in $\mathbb{R}^{d}$, submitted for publication.

13. A. Zygmund, Trigonometric Series, 2nd ed., Cambridge Univ. Press, Cambridge, 1968.

Department of Mathematics, University of Oregon, Eugene, Orggon 97403, U.S.A.

E-mail: yuan@math.uoregon.edu 